\title{
Licenciatura em matemática e o percurso de estudo e pesquisa: uma proposta do modelo epistemológico de referência para o ensino e aprendizagem do conceito de função
}

Mathematics Degree and the study and research course: a proposal of the epistemological model of reference for teaching and learning the concept of function

Rochelande Felipe Rodrigues

Marcus Bessa de Menezes ${ }^{2}$

Marcelo Câmara dos Santos ${ }^{3}$

\section{Resumo}

O referido trabalho é sobre o Percurso de Estudo e Pesquisa (PEP), um dispositivo didático que pode ser utilizado como metodologia para o ensino e aprendizagem dos conceitos matemáticos nas licenciaturas em Matemática. O PEP tem como base teórica a Teoria Antropológica do Didático (TAD), tendo Yves Chevallard como o seu principal representante. Também utilizamos a noção de Contrato Didático (CD) de Guy Brousseau, que traz elementos importantes de análise dos encaminhamentos do PEP, ressaltando as rupturas no processo. A proposta de aplicação do PEP será por meio do conteúdo de Função, um importante conteúdo da Educação Básica e da Educação Superior. O trabalho apresenta alguns pontos de ligação entre $O C D$ e o PEP, que podem identificar algumas dificuldades na implementação do dispositivo didático, principalmente na mudança de contrato didático já estabelecido para um novo contrato, dificultando as possíveis rupturas e ocasionando outras dificuldades. Outro ponto é a utilização do PEP para apresentar outra maneira de trabalhar os conceitos matemáticos nas licenciaturas em Matemática.

Palavras-chave: Teoria Antropológica do Didático; Percurso de Estudo e Pesquisa; Ruptura de Contrato Didático; Função.

\footnotetext{
${ }^{1}$ Aluno de doutorado do Programa de Pós-Graduação em Ensino das Ciências da Universidade Federal Rural de Pernambuco - UFRPE | felipemtm@gmail.com

2 Professor da Universidade Federal de Campina Grande - UFCG | marcusbessa@gmail.com

3 Professor da Universidade Federal de Pernambuco - UFPE | marcelocamaraufpe@yahoo.com.br
} 


\section{Abstract}

This work is about the Study and Research Course (PEP) as a didactic device that can be used as a methodology for teaching and learning mathematical concepts in the Mathematics Degree. The PEP has as theoretical basis the Anthropological Theory of Didactics (TAD), with Yves Chevallard as its main representative, as well as Guy Brousseau's Notion of Didactic Contract (CD), which contains important elements of PEP referral analysis, Highlighting the ruptures in the process. The proposal to apply the PEP will be through the content of Function, an important content of Basic Education and Higher Education. The paper presents some points of connection between the CD and the PEP, which can identify some difficulties in the implementation of the didactic device, mainly in the change of didactic contract already established for a new contract, making it difficult the possible ruptures and causing other difficulties. Another point is to use the PEP to present another way of working mathematical concepts in mathematics degrees.

Keywords: Anthropological Theory of Didactics; Course of Study and Research; Rupture of Didactic Contract; Function.

\section{Introdução}

O processo de ensino dos conceitos matemáticos em qualquer nível tem a sua finalidade direcionada para a aprendizagem, tal processo vem alicerçado por propostas metodológicas de ensino que buscam a compreensão dos conceitos matemáticos de maneira representacional, estrutural e funcional. Nesse processo, as propostas metodológicas têm a finalidade de representar, organizar e aplicar os conceitos buscando a compreensão do seu papel na sociedade, isto é, apresentando um sentido para os conceitos estudados. Davis e Hersh (1985) destacam a construção do sentido e da importância da utilização da Matemática para o desenvolvimento da sociedade, ocasionando em avanços conceituais em diferentes áreas, como, por exemplo, as áreas ligadas à evolução dos computadores.

Essa demanda de buscar um sentido na aplicação dos conceitos matemáticos nas diferentes instituições de ensino foram pontos discutidos na Comissão Internacional para a Instrução Matemática, acontecida no Kuwait em 1986, ficando evidente a inclusão do estudo de suas aplicações. Nesse sentido, as instituições responsáveis pelo processo de ensino e aprendizagem da Matemática devem refletir sobre a condução que é seguida atualmente, se realmente estão preparando os alunos e futuros professores para compreender os conceitos matemáticos e suas aplicações.

As instituições de ensino superior, mais especificamente nos cursos de licenciatura em Matemática, são locais de formação de professores que devem estar alinhados com as diretrizes curriculares nacionais, com o intuito de construir práticas de ensino que irão influenciar diretamente na formação inicial e continuada. A Sociedade Brasileira de Educação Matemática (SBEM, 2013) destaca a finalidade dos cursos de licenciatura em Matemática na preparação dos futuros professores, nos aspectos conceituais e didáticos, buscando a compreensão dos conceitos matemáticos e a sua aplicação, dando sentido ao campo educativo e relacionando com os possíveis problemas do cotidiano.

Onuchic e Allevato (2009) comentam que os professores das licenciaturas em Matemática estão concluindo os seus cursos mal preparados para enfrentar as salas de 
aulas da Educação Básica. Parra e Otero (2011) ressaltam que o modelo atual das universidades apresenta um autismo institucional ${ }^{4}$, tanto pelos seus integrantes, como também pela sociedade. Esse autismo está diretamente ligado às metodologias adotadas nos cursos de licenciatura em Matemática, dificultando a evolução da compreensão dos conceitos, assim como a sua funcionalidade. Reforçando essa ideia, Fonseca, Bosch e Gascón (2007) comentam que os conceitos matemáticos são estudados de uma única maneira, pontual e pouco articulados, refletindo esse problema nos diferentes níveis de ensino.

Outro problema na formação é a epistemologia escolar monumentalista ${ }^{5}$, presente na educação básica no sentido em que os conteúdos são visitados durante a formação, não compreendendo a finalidade e aplicação dos conceitos (CHEVALLARD, 2006). Nos cursos de licenciatura em Matemática a possível reprodução de conteúdos sem uma compreensão real da sua verdadeira finalidade é repassada para o futuro professor em sua prática docente, fazendo com que ele ressalte as técnicas de resolução em problemas já determinando os caminhos de respostas. Esse direcionamento pode ocasionar em uma "fraude epistemológica" conforme D'Amore (2007):

\begin{abstract}
O aluno produz uma resposta correta, mas não porque tenha entendido a sua necessidade matemática ou lógica a partir do enunciado, não porque tenha "compreendido e resolvido o problema", não porque tenha aprendido um objeto matemático, mas simplesmente porque estabeleceu uma semelhança com outro exercício; ele apenas reproduziu uma solução já feita por outros para ele (D'AMORE, 2007, p. 191).
\end{abstract}

Na formação do professor de Matemática surge a necessidade de discutir novos caminhos metodológicos, buscando a compreensão dos conceitos estudados e de sua funcionalidade.

Esse artigo tem a finalidade de discutir sobre um dispositivo didático chamado de Percurso de Estudo e Pesquisa (PEP), que servirá de base para uma aplicação prática em uma disciplina de Matemática básica da licenciatura em Matemática, utilizando o conceito de função. O PEP têm alguns elementos conceituais que fazem parte do dispositivo, como, por exemplo, o modelo epistemológico de referência (MER) e os tipos de organizações matemáticas (OM) que serão apresentados mais adiante. Com a finalidade de analisar o processo de aplicação do PEP, utilizaremos a noção de contrato didático (CD) observando as possíveis evoluções do dispositivo. O dispositivo vem como uma proposta metodológica para cursos de licenciatura em Matemática, apresentando-se com a finalidade de compreender a razão de ser dos conceitos matemáticos.

\footnotetext{
${ }^{4} \mathrm{O}$ sentido do autismo institucional das universidades está ligado a uma deficiência na sua comunicação com a sociedade.

5 Termo utilizado por Chevallard, que usa o sentido de monumento, onde são visitados, observados e apreciados, mas depois de um curto espaço de tempo as características são esquecidas. Nesse sentido, faz-se a semelhança com os conceitos matemáticos estudados na escola.
} 


\section{Alguns Elementos da Teoria Antropológica do Didático}

As discussões teóricas e metodológicas ligadas a Didática da Matemática estão direcionadas, dentre outras questões, à construção de modelos didáticos ${ }^{6}$ ligados ao ensino e aprendizagem da Matemática dos diferentes níveis de ensino, em que uma noção ou teoria pode auxiliar a outra. Destacamos em nossa discussão a Teoria Antropológica do Didático e alguns elementos do Contrato Didático que trazem contribuições importantes na construção e análise dos modelos propostos, a exemplo do PEP.

Segundo Almouloud (2007), a TAD é uma contribuição importante para a Didática da Matemática, apresentando elementos que esclarecem a discussão relacionada ao ensino e à aprendizagem das organizações matemáticas, analisando objetos existentes no sistema didático ${ }^{7}$.

Quanto à TAD, Chevallard (1996, p. 127) propõe inicialmente três conceitos primitivos: os objetos O, as pessoas X, as instituições I. Chevallard (1996) chama atenção para os objetos $O$, por ser necessário e fundamental, considerando que "todas as coisas são objetos". Ou seja, o objeto irá existir caso seja reconhecido por, pelo menos, uma pessoa X ou instituição I (BESSA MENEZES, 2010). A definição de pessoa proposta por Chevallard não deve generalizar o fato de que "todo indivíduo é uma pessoa". Pessoa surge das várias relações que o indivíduo tem com instituições diferentes, ou seja, é o conjunto de sujeitos do indivíduo é que forma a pessoa. Para o pesquisador, a pessoa muda pelas sujeições que são incorporadas com o tempo, pelo qual a relação pessoal com o objeto promove a aprendizagem. Dependendo da mudança e da evolução de suas relações pessoais com os objetos, o indivíduo permanece invariante (ARAÚJO, 2009, p. 37). O conceito primitivo de Instituição é conceituado por Chevallard (1996, p. 129) "[...] a instituição pode ser quase tudo o que quer que seja. Uma escola é uma instituição, tal como o é uma sala de aula; mas existe igualmente a instituição <trabalhos orientados>, a instituição <curso>, a instituição <família>". Araújo (2009) exemplifica que a sala de aula e o estabelecimento escolar são instituições do sistema educativo, onde impõe aos seus sujeitos suas regras, condicionando a maneiras próprias de fazer e de pensar.

Outro elemento da teoria é a noção de praxeologia apresentada por Chevallard (1996). A praxeologia é composta por certo tipo de tarefas ( $T$ ) que é conduzida por emprego de uma ou mais técnicas $(\tau)$, constituindo um bloco prático-técnico (o saber fazer). As técnicas são amparadas por uma tecnologia $(\theta)$ e justificada por uma teoria $(\Theta)$, bloco tecnológicoteórico (o logos). Como exemplo, temos a seguinte tarefa: Determine o zero da função $f(x)=3 x-1$. Para resolver essa tarefa podemos utilizar a seguinte técnica: primeiramente consideramos a igualdade $f(x)=0$ e substituímos na função ficando com a seguinte equação, $3 x-1=0$, resolvendo: $3 x-1+1=0+1$ (somar +1 nos dois lados da igualdade), $3 x=1 \therefore 3 x \cdot \frac{1}{3}=1 \cdot \frac{1}{3}$ (multiplicar $\frac{1}{3}$ nos dois lados da igualdade), obtendo

\footnotetext{
${ }^{6}$ Um modelo didático está relacionado a um modelo epistemológico, no caso, ligado a uma conceptualização do que se entende do que é ensinar e aprender Matemática, nos momentos históricos, culturais e tradicionais (BOSCH e GASCÓN, 2010).

${ }^{7}$ Chevallard (2009a) caracteriza um sistema didático como uma reunião de três instâncias: estudante, professor e um desafio didático, no qual faz com que o estudante tente resolver o desafio didático por intermédio do professor.
} 
$x=\frac{1}{3}$ como o zero da função. Essa técnica utilizada tem como justificativa tecnológica $(\theta)$ as propriedades da equação e é alicerçada pelas teorias $(\Theta)$ da álgebra.

Notamos que qualquer tipo de tarefas vem acompanhado pelos blocos prático-técnico e o tecnológico-teórico, mas na formação inicial dos professores de Matemática existe uma forte tendência em abordar prioritariamente as tarefas e as técnicas sem relacionar com a tecnologia e a teoria, retirando o sentido da finalidade dos conceitos matemáticos abordados na sua formação.

Nas praxeologias duas organizações estão presentes: a organização matemática (OM) que está relacionada à construção Matemática ligada às situações didáticas; e as organizações didáticas (OD), são organizações que fazem a transposição das OM com a finalidade do ensino e aprendizagem. O conjunto de organizações (OM e OD) permite analisar a prática durante as situações didáticas.

As diferentes praxeologias são utilizadas para facilitar a análise do processo didático, verificando a complexidade crescente das organizações matemáticas (CHEVALLARD, 1999). Tais praxeologias foram definidas em Organizações (ou praxeologias) Matemáticas pontuais, locais, regionais e global. A organização matemática pontual (OMP) é centrada em uma única técnica para ser utilizada a um único tipo de tarefas, neste caso está definida inicialmente a partir do bloco prático-técnico, apesar de que a tarefa está fundamentada por uma técnica $(\tau)$, pela tecnologia $(\theta)$ e pela teoria $(\Theta)$. A organização matemática local (OML) é composta pela interação de várias OMP, validadas e justificadas por uma tecnologia com a finalidade de fundamentar as OMP que fazem parte da OML. A organização matemática regional $(\mathrm{OMR})$ é composta pelo conjunto das $\mathrm{OML}$, cuja uma teoria $(\Theta)$ fundamenta, coordena e integram as $\mathrm{OML}$ em um discurso teórico matemático comum. Por último, a organização matemática global (OMG) composta por OMR agregando várias teorias $\left\{\Theta_{1}, \Theta_{2}, \Theta_{3}, \ldots, \Theta_{\mathrm{n}}\right\}(L U C A S, 2015)$.

A crescente complexidade das OM tem a proposta de conduzir o aluno a uma mudança de suas praxeologias, fazendo com que ele possa resolver tarefas mais complexas. Ao apresentarmos a seguinte tarefa: construir o gráfico da função $f(x)=3 x-2$, nesse caso temos uma OMP, que apresenta um tipo de tarefa e consequentemente a uma técnica. Quando temos tipos de tarefas que utilizam técnicas diferentes, mas que são justificadas por uma tecnologia, por exemplo: Resolva analiticamente e graficamente o sistema de equações $\left\{\begin{array}{c}x-y=-3 \\ 2 x+3 y=4^{\prime}\end{array}\right.$, nesse caso será preciso utilizar técnicas diferentes para resolver. Analiticamente podem ser utilizadas as diferentes técnicas de resolução de sistema de equações, como, por exemplo, o método da adição, da substituição, por escalonamento, entre outros. Graficamente, a utilização do sistema cartesiano auxiliado pelas técnicas de construção gráfica presente no conteúdo de função, nos dão uma visualização do sistema no plano cartesiano. A utilização dessas técnicas é justificada pelo sistema de equações lineares e representada pelo sistema cartesiano. Ao utilizarmos um conjunto de OML teremos a construção da OMR, no nosso exemplo, representa uma OML das várias que devem ser desenvolvidas, constituindo uma OMR justificada pela teoria da álgebra linear. Consequentemente além da teoria da álgebra linear, juntamente com as teorias da álgebra elementar, álgebra abstrata, álgebra computacional e outras que fazem parte do ramo da Matemática denominada álgebra, constituindo em uma OMG.

Para um direcionamento e análise das aplicações das organizações matemáticas Chevallard $(1997,1999)$ apresenta seis momentos para descrever as OD, ressaltando que 
eles podem acontecer de maneira simultânea. Esses momentos dão o sentido didático das praxeologias que são utilizadas e desenvolvidas pelos alunos e encaminhadas pelo professor.

O primeiro momento é o encontro com as organizações matemáticas (OM) propostas. Essas OM estão ligadas com a influência cultural e de suas práticas sociais, como também, a escolha do professor das situações fundamentais (Brousseau, 1986). As situações fundamentais proporcionam aos alunos o sentido dos conceitos estudados, fazendo com que os alunos sejam os atores principais do processo didático, com a finalidade do desenvolvimento das OM. O segundo momento é o da exploração dos tipos de tarefas e da elaboração de técnicas. Esse momento nos permite criar e usar uma técnica relativa a problemas do mesmo tipo, ou seja, a elaboração das técnicas é um meio para resolver de maneira quase rotineira estes problemas. O terceiro momento de estudo é o da constituição do entorno tecnológico-teórico relativo à técnica proposto pela $O M$, relacionado diretamente com os momentos anteriores, com a finalidade de fundamentar e justificar as técnicas utilizadas. O quarto momento é o momento de trabalho da técnica, a utilização da técnica de fato, proporcionando a sua validação ou adequação. O quinto momento é o da institucionalização, ressaltando qual a OM construída, explicitando os elementos que fazem parte de sua constituição. E, por último, o momento da avaliação, ligado diretamente ao momento da institucionalização, no sentido de que o processo é avaliado no que se refere aos momentos pontuais e no seu contexto geral, fazendo um balanço dos momentos (CHEVALLARD, 1997, 1999).

Diante desses momentos podemos levantar dois pontos que merecem ser observados. O primeiro ponto está relacionado a situação fundamental (BROUSSEAU, 1986), discutida no primeiro momento e o segundo ponto a devolução ${ }^{8}$, ressaltando o papel do aluno por intermédio do professor. Tais pontos trazem reflexões ligadas à condução das construções das OM e das OD propostas, no qual o professor tem um papel importante na construção da situação fundamental, fazendo com que o aluno realce a sua participação, tornando-se o autor principal.

\section{Modelo Epistemológico de Referência e o Percurso de Estudo e Pesquisa}

$\mathrm{Na}$ condução das situações didáticas propostas, outro elemento presente na TAD procura dar sentido e sinalização para os caminhos de ensino e aprendizagem da Matemática, tais caminhos são alicerçados e direcionados por modelos de referência que orientam as conduções didáticas e matemáticas. Esses modelos vêm buscar uma emancipação frente a epistemologia dominante ${ }^{9}$ presente nas instituições de ensino, no qual o monumentalismo está presente de maneira marcante.

\footnotetext{
${ }^{8}$ A maneira de comunicar um problema ao aluno faz com que ele se sinta o único responsável a respondê-lo, isto é, ele torna o problema seu aceitando a responsabilidade na condução da resposta, fazendo com que ele tenha um desenvolvimento autônomo (BROUSSEAU, 1996).

${ }^{9}$ É a forma concreta em que as universidades como instituições docentes, mas concretamente, a comunidade de agentes interverem nos processos de estudos das matemáticas, fazendo com que os professores universitários e os estudantes interpretam a matemática e a sua relação com a instituição (Barquero, Bosch e Gascón, 2010).
} 
O modelo a ser apresentado inicialmente é o modelo epistemológico de referência (MER), um modelo cuja organização matemática, seguida de uma organização didática apresenta-se como alternativa frente à epistemologia dominante na instituição de ensino. Gascón (2014) destaca que "para ter os processos de transposição didática como objeto de estudo, a didática necessita analisar de maneira crítica os modelos epistemológicos das matemáticas dominantes nas instituições envolvidas, e assim libertar-se da falta de crítica desses modelos" (Gáscon, 2014, p. 100, tradução nossa). Tal enfrentamento é materializado pelo MER, no qual a transposição didática conduz para a modelização matemática da OM de um MER e os seus ajustes na OD, juntamente com o estudo crítico dos documentos oficiais, das concepções dos professores e alunos, dos livros textos, dos planos de estudos, das praxeologias atuais e entre outros, fazem parte de sua construção.

O MER pode ser específico ou geral. No caso do específico está relacionado a temas matemáticos como, por exemplo, divisibilidade elementar (Gascón, 2001); limite de funções (Barbé et al., 2005); proporcionalidade no âmbito das relações funcionais elementares (García et al., 2006); sistema de numeração (Sierra et al., 2007); modelização matemática (Barquero et al., 2011; Serrano et al., 2010) e modelização algébrico-funcional (Ruiz-Munzón et al., 2011). No caso do geral, estão ligados a estruturas mais amplas que podem englobar os específicos, tais como a caracterização do modelo epistemológico dominante da atividade Matemática no ensino secundário da Espanha (Fonseca et al., 2004); reformulação do problema da metacognição no âmbito da TAD (Rodríguez et al., 2007). (LUCAS, 2015).

Em um entendimento mais amplo dos MER, temos os modelos didáticos de referência (MDR) que estão direcionados a um sistema didático formado por uma instituição mandante com uma estrutura consistente, em que apresenta os desafios crescentes de estudos, com a constituição de grupos de alunos e professores que buscam as respostas dos desafios propostos. Segundo Bosch e Gascón (2010), os MER e MDR:

Em particular devem servir para questionar, analisar e avaliar (em lugar de aceitar acriticamente) os dois tipos de modelos dominantes nessas instituições: por outro lado, os modelos epistemológicos das matemáticas que se tomam como transparente e indiscutível nas instituições tidas como "nobres" pelo seu prestígio e legitimidade social, aparecem como responsáveis por moldar o "saber sábio" (mas não desejam fazer parte do objeto de estudo da didática), por outro lado os modelos de estudos mais ou menos espontâneos que constituem a forma culturalmente de aceitar e interpretar o ensino e a aprendizagem escolar da matemática, consequentemente, tem grande preponderância nas instituições de ensino (BOSCH e GASCÓN, 2010, p. 62) (tradução nossa).

Observamos que os MER e os MDR, apresentam elementos importantes frente aos possíveis problemas relacionados à epistemologia dominante presente nas instituições de ensino. Diante do enfrentamento da epistemologia dominante presente nos cursos de licenciatura em Matemática, nossa discussão direciona para o Percurso de Estudo e Pesquisa (PEP), que foi inserido na sua metodologia o MER e o MDR para realizar os tipos de PEP. Esse dispositivo é um tipo de engenharia didática ${ }^{10}$ ligada à organização didática e à modelização matemática para o ensino e aprendizagem.

\footnotetext{
${ }^{10}$ Para melhor compreensão observar Almouloud e Silva (2012) e Chevallard (2009b).
} 
Chevallard (2004, 2006, 2009a) apresenta um dispositivo didático direcionado à modelização matemática ${ }^{11}$, chamado de Percurso de Estudo e Pesquisa (PEP), no qual a sigla PEP foi legitimada no Brasil por Chevallard, em 2011. O PEP está relacionado a um estudo de uma questão viva $\left(Q_{0}\right)$ ou questão geratriz, isto é, uma problemática relevante que pode gerar outras problemáticas menores derivadas dela $\left(Q_{i}\right)$. Tal problemática $\left(Q_{0}\right)$ e suas derivações $\left(Q_{i}\right)$, conduzem a um grande número de saberes que possibilitará caminhos de resoluções diferentes $\left(R_{i}\right)$ e a verificação de suas limitações nas conduções desses caminhos. Nessa cadeia de questões $\left(Q_{i}\right)$ são geradas cadeias de respostas $\left(R_{i}\right)$, que terá a seguinte representação $\left(Q_{i}, R_{i}\right)$.

Segundo Fonseca, Casas, Bosch e Gascón (2009, pg. 2), o "[...] O PEP vem recuperar a genuína relação entre perguntas e respostas que está na origem da construção do conhecimento científico em geral e das atividades de modelização em particular". Chevallard (2009a) apresenta um modelo representado pelo "esquema herbatiano" ${ }^{\prime 2}$ de maneira condensada:$$
(S(X ; Y ; Q) \rightarrow M) \rightarrow R^{\vee}
$$

E pela sua representação expandida:

$$
\left[S(X ; Y ; Q) \rightarrow\left\{R_{1}^{0}, R_{2}^{0}, \ldots, R_{n}^{0}, O_{n+1}^{0}, \ldots, O_{m}^{0}\right\}\right] \rightarrow R^{\bullet}
$$

No modelo proposto, temos $S(X ; Y ; Q)$ que é caracterizado por um sistema didático formado por um grupo $Y$ (professor, orientador, coordenador de pesquisa, entre outros) que deve fazer, estudar, reconstruir e deixar acessíveis os caminhos possíveis para um grupo de alunos $X$, na busca de responder uma questão geratriz $Q_{0}$, cujo o estudo conduza para as reconstruções dos principais elementos da organização matemática local (OML) de partida. O sistema $\left\{R_{1}^{0}, R_{2}^{0}, \ldots, R_{n}^{0}, O_{n+1}^{0}, \ldots, O_{m}^{0}\right\}$ representa o conjunto de recursos que servirá para produzir a resposta final $R^{\star}$. Nesse modelo, propõe que, durante 0 desenvolvimento do estudo será mobilizado todos os recursos médios ${ }^{13}$, saberes e respostas disponíveis $R_{i}^{\diamond}$, para construir uma boa resposta $R^{\bullet}$, na qual os objetos de qualquer natureza $O^{\diamond}$ atuarão como médio para colocar em prova as $R_{i}^{\diamond}$. Lucas (2015, p. 33), comenta "que o esquema herbartiano pode ser considerado um sistema de referência didático para observar, descrever, analisar e avaliar os sistemas existentes nas instituições sociais ou teoricamente possíveis. Ele fornece um modelo geral entendida pela TAD «estudar uma questão»" (tradução nossa).

Rodríguez, Bosch e Gascón (2007) comentam que o PEP surge a partir de questões problemáticas, as quais, em suas resoluções, necessitam de construções de várias praxeologias, nesse caso, um conjunto de conhecimentos teóricos e práticos articulados

\footnotetext{
${ }^{11}$ A TAD descreve a Modelização Matemática como um processo de construção e reconstrução das OM complexas e crescentes (OMP, OML, OMR), partindo de uma questão problemática que constitui a razão de ser do processo (LUCAS, 2015).

${ }^{12} \mathrm{O}$ esquema herbatiano está melhor detalhado em Chevallard (2009b).

${ }^{13}$ Instrumento indispensável para colocar à prova as respostas geradas a partir das $R_{i}^{\natural}$, verificando a sua validez (BOSCH e GASCÓN, 2010). É importante destacar que o sentido do médio vai além do apresentado no texto, no qual podemos associar ao termo "milieu" discutido por Brousseau.
} 
entre si. Desse modo, no PEP, a questão geratriz aparecerá como possibilidade de deixar mais viva a resolução de um problema em sala.

As considerações de Rodríguez, Bosch e Gascón (2007), ressaltam que as possibilidades de aplicações de um PEP e de sua eficácia na incorporação de resoluções de problemas no processo de ensino e de aprendizagem, está ligada a uma nova partição de responsabilidades definidas pelo contrato didático:

\begin{abstract}
Estabelece-se então uma relação que determina - explicitamente em pequena parte, mas sobretudo implicitamente - aquilo que cada parceiro, o professor e o aluno, tem a responsabilidade de gerir e pelo qual será, de maneira ou de outra, responsável perante o outro. Este sistema de obrigações recíprocas assemelha-se a um contrato. Aquilo que aqui nos interessa é o contrato didático, ou seja, a parte deste contrato que é específica do conteúdo: o conhecimento matemático visado (BROUSSEAU, 1996, p. 51).
\end{abstract}

Essas responsabilidades são compartilhadas entre professores e alunos de forma equilibrada. Considerando tais reflexões, podem ser propostas duas questões ligadas à formação dos licenciandos em Matemática: 1) O que dificulta este compartilhamento de responsabilidades entre professor e alunos? 2) O que dificulta ao aluno assumir as responsabilidades que venham a ser atribuídas na resolução do problema proposto ou construído em sala?

A aplicação do PEP exige mudança em relação a algumas normas que são impostas por níveis maiores ao disciplinar que, por essa razão, pode se converter em restrições para ser posta em prática. Chevallard (2007), com a finalidade de estudar e compreender as influências e restrições nas OM e OD apresenta os níveis de codeterminação, podendo assim reestruturar e reorganizar as condições de ensino e aprendizagem.

\title{
Civilização \\ $\downarrow \uparrow$ \\ Sociedade \\ $\downarrow \uparrow$ \\ Escola \\ $\downarrow \uparrow$ \\ Pedagogia \\ $\downarrow \uparrow$ \\ Disciplina \\ $\downarrow \uparrow$ \\ Domínio \\ $\downarrow \uparrow$ \\ Setor \\ $\downarrow \uparrow$ \\ Tema \\ $\downarrow \uparrow$ \\ Tópico
}

Figura 01: Níveis de Coderterminação. Fonte: Chevallard, 2007 
Rodríguez, Bosch e Gascón (2007, p. 488) afirmam que "[...] Os PEP que não se mostram centrados em um tema ${ }^{14}$, setor, domínio, nem sequer disciplina, encontra dificuldades para serem desenvolvidas dentro de uma aula normal de Matemática".

O PEP propõe uma nova maneira de observar os problemas de ensino e aprendizagem da Matemática, partindo de um tema central ou questão geratriz e, a partir daí, iniciar todo o processo de desenvolvimento na busca de uma boa resposta, uma resposta não rotulada ${ }^{15}$, mas construída a partir das articulações de conhecimentos adquiridos no processo. Nesse sentido o contrato didático (BROUSSEAU, 1996), emerge como elemento importante a ser analisado, pois para uma mudança de postura do professor e dos alunos mediados pelo saber em questão poderá efetivar uma ruptura no contrato vigente ${ }^{16}$, refletindo no surgimento de novas praxeologias.

Nesse sentido, o que são analisadas são as relações implícitas ou explícitas que se estabelecem no contrato didático e de que modo elas se manifestam, efetivando rupturas, ou não, das regras anteriormente estabelecidas. Brousseau (1986) comenta que o mais importante não é tentar explicitar a totalidade das regras que constituem o contrato didático, mas delinear alguns pontos de suas possíveis rupturas. No desenvolvimento do PEP, em momentos em que as OM são mudadas por outras mais complexas, existe a possibilidade de identificar as possíveis rupturas de contrato com a finalidade de analisar a evolução do PEP.

As rupturas de contrato são elementos importantes no processo de ensino e aprendizagem da Matemática, pois promovem a possível compreensão dos conceitos trabalhados pelo professor. Segundo Araújo, Brito Lima e Câmara (2011), tais conceitos a serem trabalhados na forma de atividades diferentes das atuais promovem uma tensão entre professores e alunos, fazendo com que eles utilizem novas formas de conduzir as suas respostas, ocasionando as rupturas de contrato. Portanto, podemos considerar a afirmação de Sarrazy (1995, p. 6): "De fato, a aprendizagem vai repousar não sobre o funcionamento do contrato, mas sobre suas rupturas". No momento de aplicação do PEP, o contrato didático pode apresentar o que realmente foi ou está sendo modificado na prática do professor, assim como as possíveis compreensões do aluno frente ao saber.

Após discutirmos sobre o PEP e de alguns elementos teóricos que fazem parte de sua constituição, daremos continuidade apresentando uma proposta do modelo epistemológico de referência para o conceito de função que está na fase inicial de construção, no qual servirá de base para uma aplicação futura de um PEP a alunos de licenciatura em Matemática.

\footnotetext{
${ }^{14}$ Tema, setor, área e disciplina da matemática.

${ }^{15} \mathrm{Na}$ perspectiva do PEP, a resposta rotulada é aquela que já se encontra pronta ou quase pronta desde o início da questão geratriz.

${ }^{16}$ No contrato didático existem regras implícitas e explicitas no jogo didático, no momento em que essas regras são quebradas, isto é, as expectativas do aluno ou do professor não são concretizadas gerando tensões, emergem as rupturas de contrato (Brousseau, 1996).
} 


\section{Proposta de um PEP para o ensino de Função}

O curso de licenciatura em Matemática é composto por vários conteúdos que devem ser ensinados de maneira articulada, partindo da compreensão específica até a geral, interligando com o seu papel na sociedade (MINISTÉRIO DA EDUCAÇÃO, 2002).

O conteúdo de função e de suas funções elementares está presente nas disciplinas que antecedem as disciplinas de cálculo, denominadas nos currículos de Pré-cálculo, Matemática Básica, Fundamentos da Matemática ou equivalente. A sua compreensão conceitual e de sua aplicação é fundamental para as disciplinas de cálculo.

O conceito de Função é um dos mais importantes a serem aplicados no ensino médio, como também no ensino superior, pois está presente em vários ramos da Matemática, como também em aplicações voltadas para o cotidiano (BRASIL, 2006; EVES, 2004).

Para a aplicação da nossa proposta utilizando o PEP, é necessário a construção de um modelo epistemológico de referência direcionado para o estudo de função, trazendo elementos básicos para o seu desenvolvimento. Para a constituição desse MER será utilizado os quatro estágios de modelização Matemática (Chevallard, 1989, Gascón, 2001):

$1^{\circ}$ Estágio: Delimitação ou construção do sistema a modelizar que formula questões problemáticas;

$2^{\circ}$ Estágio: Construção dos modelos e formulação das questões iniciais;

$3^{\circ}$ Estágio: Utilização do modelo e sua interpretação;

$4^{\circ}$ Estágio: Novo processo de modelização.

Auxiliando nessa construção os três níveis de modelização algébrico-funcional (RUIZMUNZÓN, 2010):

$1^{\circ}$ Nível: funções isoladas de uma única variável;

$2^{\circ}$ Nível: famílias de função de uma variável;

$3^{\circ}$ Nível: famílias de funções de duas ou mais variáveis.

Além dos quatro estágios de modelização matemática e dos níveis de modelização algébrico-funcional, levará em conta alguns elementos apresentados por Lucas (2015), como também um levantamento documental e de entrevistas para identificar e compreender os elementos presentes na epistemologia dominante na condução do conceito de função.

Para análise, teremos a Teoria Antropológica do Didático na identificação e estudo das praxeologias dos professores e a noção do contrato didático, observando as modificações na condução do saber através da identificação de suas rupturas de contrato. Nesse sentido, pretendemos responder à seguinte pergunta: como compreender as restrições e promover as condições necessárias para que o Percurso de Estudo e Pesquisa possa ser conduzido no processo de ensino e aprendizagem do conceito de função trabalhado no curso de licenciatura em Matemática?

\section{Considerações Finais}

Nossa discussão é sobre um dispositivo didático chamado de Percurso de Estudo de Pesquisa (PEP), que está fundamentado teoricamente no campo da Didática da Matemática e tem com a Teoria Antropológica do Didático a sua base conceitual. Contudo, a noção de contrato didático tem um papel importante no auxílio da compreensão dos fenômenos que um PEP poderá proporcionar, tanto nas mudanças desse contrato, bem como em suas rupturas. 
O PEP apresenta uma proposta metodológica diferente da vigente nos cursos de licenciatura em Matemática, indicando "novos" caminhos para o professor buscar o ensino dos conceitos previstos durante o curso, o que poderá promover uma compreensão mais significativa desses conceitos pelos alunos. Nesse processo de percurso, existe a possibilidade de aplicação para resolver problemas relacionados à realidade do aluno ou à sociedade na qual ele está inserido, buscando apresentar-se como uma resposta aos problemas relacionados a epistemologia escolar monumentalista.

A utilização do PEP requer mudanças na prática docente e, consequentemente, nas ações dos alunos em sala. Essas mudanças tendem a promover rupturas no processo didático que envolve professor-alunos-saber. O papel do professor em incluir tipos de tarefas diferentes que envolvam técnicas diferentes, partindo de uma organização pontual para uma local; local para regional e regional para global, pode favorecer uma ampliação do conceito trabalhado, mobilizando mais de uma técnica e mais de uma tecnologia. Nesse processo, o contrato didático, a situação fundamental e a devolução são elementos a serem observados na condução do dispositivo, trazendo pontos relacionados à postura do professor e a atuação do aluno frente ao saber.

Para a nossa proposta de aplicação do PEP utilizando o conceito de função em uma disciplina da licenciatura em Matemática, construiremos um modelo epistemológico de referência que servirá de base para elaboração e aplicação do dispositivo, no qual estará principalmente baseado na modelização matemática e na modelização algébrico-funcional. Durante a aplicação do PEP o contrato didático nos possibilitará observar e analisar qual o comportamento dos alunos frente ao conceito de função, observando suas rupturas, as mudanças de praxeologias e os tipos de organizações matemáticas que emergem nos momentos didáticos.

\section{Referências}

ALMOULOUD, S. A. Fundamentos da didática da matemática. Curitiba: Editora UFPR, 2007.

ALMOULOUD, S. A.; SILVA, M. J. F. Engenharia didática: evolução e diversidade. Revemat: Revista Eletrônica de Educação Matemática, Florianópolis, v. 7, n. 2, p. 22-52, 2012. Disponível em: <https://periodicos.ufsc.br/index.php/revemat/article/viewFile/19811322.2012v7n2p22/23452>. Acesso em: 06 out. 2017.

ARAÚJO, A. J. O ensino de álgebra no Brasil e na França: estudo sobre o ensino de equações do $1^{\circ}$ grau à luz da teoria antropológica do didático. 2009. 292 f. Tese (Doutorado em Educação)- Universidade Federal de Pernambuco, Recife, 2009. Disponível em: <http://repositorio.ufpe.br/bitstream/handle/123456789/3947/arquivo3433_1.pdf?sequence =1\&isAllowed=y>. Acesso em: 06 out. 2017.

ARAÚJO, L. F.; LIMA, A. P. A. B.; SANTOS, M. C. Ruptura e efeitos do contrato didático numa aula de resolução de problemas algébricos. Revista Brasileira de Estudos Pedagógicos, Brasília, v. 92, n. 232, p. 739-756, set./dez. 2011. Disponível em: <http://rbep.inep.gov.br/index.php/rbep/article/view/676/654>. Acesso em: 06 out. 2017.

BARBÉ, J. et al. Didactic restrictions on the teacher's practice: the case of limits of functions in Spanish High Schools. Educational Studies in Mathematics, [S.I.], v. 59, p. 235-268, 2005. 
Disponível em: <https://link.springer.com/article/10.1007/s10649-005-5889-z>. Acesso em: 06 out. 2017.

BARQUERO, B. Enseñando modelización a nivel universitario: la relatividad institucional de los recorridos de estudio e investigación. Bolema, Rio Claro, v. 29, n. 52, p. 593-612, ago. 2015. Disponível em: <http://www.scielo.br/pdf/bolema/v29n52/1980-4415-bolema-29-520593.pdf>. Acesso em: 06 out. 2017.

BARQUERO, B.; BOSCH, M.; GASCÓN, J. Ecología de la modelización matemática: los recorridos de estudio e investigación. In: Aportaciones de la teoría antropológica de lo didáctico: un panorama de la TAD. Barcelona: Centre de Recerca Matemática, 2011. p. 553-577.

\section{BON, C. F. Discontinuidades matemáticas y didácticas entre la Secundaria y la}

Universidad. 2004. 283 f. Tesis (Doctorado en Ingeniería Industrial)- Universidad de Vigo, Vigo, 2004. Disponível em: <http://www.atd-tad.org/wpcontent/uploads/2012/07/TESIS_en_PDF.pdf>. Acesso em: 06 out. 2017.

BOSCH, M.; GASCÓN, J. Fundamentación antropológica de las organizaciones didácticas: de los "talleres de prácticas matemáticas" a los "recorridos de estúdio e investigación". In: BOSCH, M.; GASCÓN, J. Diffuser les mathématiques (et les autres savoirs) comme outils de connaissance et d'action. Montpellier: Université de Montpellier, 2010. p. 55-91.

BRASIL. Ministério da Educação. Ciências da natureza, matemática e suas tecnologias. Brasília, DF, 2006. 140 p.

BRASIL. Parecer no 1.302, de 06 de novembro de 2001. Diário Oficial da União, Poder Executivo, Brasília, 05 mar. 2002. Seção 1, p. 15.

BROUSSEAU, G. Fondementes e Methodes de la Didactique des Mathématiques. Recherche en Didactique des Mathématiques, [S.I.], v. 7, n. 2, p. 33-115, 1986.

BROUSSEAU, G. Os Diferentes Papéis do Professor. In: PARRA, C. et al. (Org.). Didática da Matemática: reflexões psicopedagógicas. Porto Alegre: ARTMED, 1996. p. 54-79.

CHEVALLARD, Y. Arithmétique, algèbre, modélisation: étapes d'une recherche. Marselha: IREM Aix-Marseille, 1989.

CHEVALLARD, Y. Conceitos Fundamentais da Didática: as perspectivas trazidas por uma abordagem antropológica. In: BRUN, J. et al. (Org.). Didática das matemáticas. Lisboa: Instituto Piaget, 1996.

CHEVALLARD, Y. Familière et problématique, la figure du professeur. Recherche en Didactique des Mathématiques, [S.I.], v. 17, n. 3, 1997.

CHEVALLARD, Y. L'analyse des pratiques enseignantes en théorie anthropologique didactique. Recherche en Didactique des Mathématiques, [S.I.], v. 19, n. 2, p. 221-266, 1999.

CHEVALLARD, Y. Vers une didactique de la codisciplinarité: notes sur une nouvelle épistémologie scolaire. Journées de Didactique Comparée, [S.I.], p. 1-13, mai. 2004.

CHEVALLARD, Y. Steps towards a new epistemology in mathematics education. In: CONGRESS EUROPEAN SOCIETY FOR RESEARCH IN MATHEMATICS EDUCATION, 4., 2006, Barcelona. Actas... Barcelona: FUNDEMI-IQS, 2006. p. 21-30. 
CHEVALLARD, Y. Passé et présent de la théorie anthropologique du didactique. In: RUIZHIGUERAS, L.; ESTEPA, A.; GARCÍA, F. J. (Org.). Sociedad, escuela y matemáticas: aportaciones de la teoría antropológica de la didáctica. Jaén: Servicio de publicaciones de la Universidad de Jaén, 2007. p. 705-746.

CHEVALLARD, Y. La notion de PER : problèmes et avancées. Toulouse: UMF/ADER, 2009a.

CHEVALLARD, Y. La notion d'ingénierie didactique, un concept à refonder: La notion d'ingénierie didactique, un concept à refonder. In: ÉCOLE D'ÉTE DE DIDACTIQUE DES MATHEMATIQUES, 15., 2009, Clermont-Ferrand. Acté... Clermont-Ferrand: Université Clermont Auvergne, 2009b. p. 1-44.

D'AMORE, B. Epistemologia, didática da matemática e práticas de ensino. Bolema, Rio Claro, v. 20, n. 28, p. 179-205, 2007. Disponível em:

<http://www.dm.unibo.it/rsddm/it/articoli/damore/635\%20\%20Epistemologia\%20Didattica.p df>. Acesso em: 06 out. 2017.

DAVIS, J. P.; HERSH, R. A experiência matemática. Rio de Janeiro: Francisco Alves, 1985.

EVES, H. Introdução à história da matemática. Campinas: UNICAMP, 2004.

FONSECA, S.; BOSCH, M.; GASCÓN, J. El momento del trabajo de la técnica en la competación de organizaciones matemáticas: el caso de la "Regla de Ruffini". In: RUIZHIGUERAS, L.; ESTEPA A.; GARCIA, F. J. (Org.). Sociedad, escuela y matemáticas: aportaciones de la teoría antropológica de lo didáctico. Jaén: Universidad de Jaén. 2007. p. $1-15$.

FONSECA, S. et al. Diseño de un recorrido de estudio e investigación en los problemas de modelización. In: GONZÁLEZ, M. J.; GONZÁLEZ, M. T.; MURILLO, J. (Org.). Investigación en Educación Matemática.Comunicaciones de los Grupos de Investigación. Santander: SEIEM, 2009.

GARCÍA, F. J. et al. Mathematical modelling as a tool for the connection of school mathematics. The International Journal on Mathematics Education, [S.I.], v. 38, n. 3, p. 226-246, 2006.

GASCÓN, J. Incidencia del modelo epistemológico de las matemáticas sobre las prácticas docentes. Revista Latinoamericana de Investigación en Matemática Educativa, Cidade do México, v. 4, n. 2, p. 129-159, jul. 2001.

GASCÓN, J. Los modelos epistemológicos de referencia como instrumentos de emancipación de la didáctica y la historia de las matemáticas. Educación Matemática, [S.I.], v. 25, p. 99-123, marzo. 2014.

LUCAS, C. O. Una Posible "Razão de Ser" del Cálculo Diferencial Elemental en el Ámbito de la Modelización Funcional. 2015. 625 f. Tesis (Doctorado en Técnicas Matemáticas Avanzadas)- Universidad de Vigo, Vigo, 2015. Disponível em: <http://www.atdtad.org/wp-content/uploads/2016/01/Una-posible-\%C2\%ABraz\%C3\%B3n-deser\%C2\%BB-del-c\%C3\%A1lculo-diferencial-elemental2.pdf>. Acesso em: 06 out. 2017.

MENEZES, M. B. Praxeologia do Professor e do Aluno: uma análise das diferenças no ensino de equações do segundo grau. 2010. 178 f. Tese (Doutorado em Educação)- Universidade Federal Pernambuco, Recife, 2010. Disponível em: 
<http://repositorio.ufpe.br/bitstream/handle/123456789/3722/arquivo196_1.pdf>. Acesso em: 06 out. 2017.

ONUCHIC, L. R.; ALLEVATO, N. S. G. Formação de professores: mudanças urgentes na licenciatura em matemática. In: FROTA, M. C. R.; NASSER, L. (Org.). Educação Matemática no Ensino Superior: pesquisas e debates. Recife: SBEM, 2009.

PARRA, V.; OTERO, M. R. Praxeologías didácticas en la universidad y el fenómeno del «encierro»: un estudio de caso relativo al límite y continuidad de funciones. In: $\mathrm{BOSCH}$, M. et al. (Org.). Aportaciones de la teoría antropológica de lo didáctico: un panorama de la TAD. Barcelona: Centre de Recerca Matemática, 2011. p. 719-741.

RODRÍGUEZ, E.; BOSCH, M.; GASCÓN, J. Los recorridos de estudio investigación en La reformulación didáctica del problema de la metacognición. In: RUIZ-HIGUERAS, L.; ESTEPA, A. C.; GARCIA, F. J. (Org.). Sociedad, Escuela y Matemáticas: aportaciones de la teoría antropológica de lo didáctico. Jaén: Universidad de Jaén, 2007. p. 481-506.

RUIZ-MUNZÓN, N. La introducción del álgebra elemental y su desarrollo hacia la modelización funcional. 2010. 407 f. Thèse (Doctorat en Matemàtiques)- Universitat Autònoma de Barcelona, Barcelona, 2010.

RUIZ-MUNZÓN, N.; BOSCH, M.; GASCÓN, J. Un modelo epistemológico de referencia del álgebra como instrumento de modelización. In: BOSCH, M. et al. (Org.). Aportaciones de la teoría antropológica de lo didáctico: un panorama de la TAD. Barcelona: Centre de Recerca Matemática, 2011. p. 743-765.

SANTOS, M. B. S.; ALMOULOUD, S. A. O Conceito de Limite: estudo das organizações matemáticas e didáticas em livros didáticos. Perspectiva da Educação Matemática, Campo Grande, v. 7, n. 15, p. 537-572, 2014. Disponível em:

<http://seer.ufms.br/index.php/pedmat/article/view/902/582>. Acesso em: 06 out. 2017.

SARRAZY, B. Le contrat didactique. Revue Française de Pédagogie, [S.I.], n. 112, p. 85-118, juil./sept. 1995. Disponível em: <http://www.persee.fr/doc/rfp_0556-

7807_1995_num_112_1_1229>. Acesso em: 06 out. 2017.

SERRANO, L.; BOSCH, M.; GASCÓN, J. Fitting models to data: the mathematising step in the modelling process. In: SOURY-LAVERGNE, S.; ARZARELLO, F. Proceedings of the Sixth Congress of the European Society for Research in Mathematics Education. Lyon: INRP, 2010. p. 2185-2196.

SIERRA, T. A.; BOSCH, M.; GASCÓN, J. Interrelación entre lo matemático y lo didáctico en la reconstrucción escolar de los Sistemas de Numeración. In: RUIZ-HIGUERAS, L.; ESTEPA, A. C.; GARCIA, F. J. (Org.). Sociedad, Escuela y Matemáticas: aportaciones de la teoría antropológica de lo didáctico. Jaén: Universidad de Jaén, 2007. p. 359-381.

SOCIEDADE BRASILEIRA DE EDUCAÇÃO MATEMÁTICA. A formação do professor de matemática no curso de licenciatura: reflexões produzidas pela comissão paritária SBM/SBEM. Brasília, 2013. 43 p. 